\title{
Cooperativa agrícola y Caja Rural S. Isidro de Castellón
}

\author{
Antonio Marco Muñoz
}

\section{Sedes sociales y oficinas}

El Gremio de Labradores de San Isidro mantenía con el Circulo Católico de Obreros unas estrechas relaciones, lo que les permitió compartir la sede social, que inicialmente estaba situada en la calle de Enmedio, tal como recoge la revista El Obrero Católico de 1 de noviembre de 1893. Esta coincidencia de sedes la recoge el reglamento de 1896, teniendo los almacenes en el inmueble sito en la calle del Mar, n. ${ }^{\circ} 1$ y 3 (hoy calle Hermanos Bou), esquina con la calle Gobernador.

En setiembre de 1910 se decide en junta general la compra del solar, propiedad de los señores Matutano, situado en el camino del Mar, más tarde avenida Hermanos Bou, n. 33 encargándole las obras al arquitecto D. Godofredo Ros, que se culminan el 28 de enero de 1912. Este local disponía de dos naves, distintos despachos y dos viviendas. Allí estaban el almacén, los despachos que eran utilizados para las clases nocturnas, tanto para los socios agricultores como para sus hijos, un salón utilizado para la proyección de películas, distintas dependencias utilizadas por la cooperativa, así como por el personal de la UTECO; estos se trasladarían en los años treinta a las oficinas de la calle Herrero n. ${ }^{\circ}$, utilizadas también por la cooperativa para la celebración de asambleas.

El local contiguo al de la cooperativa, en la avda. Hermanos Bou, estaba cedido a la Sociedad de Exportación n. ${ }^{\circ} 1$ de la CASI (Cooperativa Agrícola San Isidro). Separado por la calle Marqués de la Ensenada estaba el almacén de la Cooperativa Agrícola El Litoral.

Enfrente, en el n. 34 de la avda. Hermanos Bou, se encontraba el almacén de la Sociedad de Exportación n. 3 de la CASI. En el inmueble contiguo en el que tuvo su domicilio la Cooperativa de Servicios Informáticos Infocampo (hoy ocupado por la compañía ONO), tenía su almacén la UTECo y la Unión de Productores de Almendra.

El domicilio social de la cooperativa se traslada en julio de 1930 a la calle González Cherma n. ${ }^{\circ} 63$ (hoy calle Enmedio).

El 16 de febrero de 1930 el Diario de Castellón anuncia que el Sindicato de San Isidro proyecta adquirir un local para su centro recreativo y caja de ahorros, conformado por dos salones unidos por una puerta en la parte de atrás, habiendo sido la sede de la Unión Patriótica, situados en la calle Enmedio n. 49 y 51, propiedad de los hermanos Peña y de las hermanas Rodríguez, con una superficie aproximada de 500 metros cuadrados, que los ceden en régimen de alquiler. Los citados locales tenían su entrada independiente por la calle Enmedio, con puerta posterior a la calle Maestro Chapí. Ambos locales se comunicaban interiormente por una puerta o hueco abierto en la pared medianera. Pero no fue hasta el 6 de setiembre de 1968 cuando cuajaron las ofertas de compraventa de los locales, gracias a la intervención del agente de la propiedad inmobiliaria D. Vicente Hernández Ripollés, hecho que se materializó en la notaria 


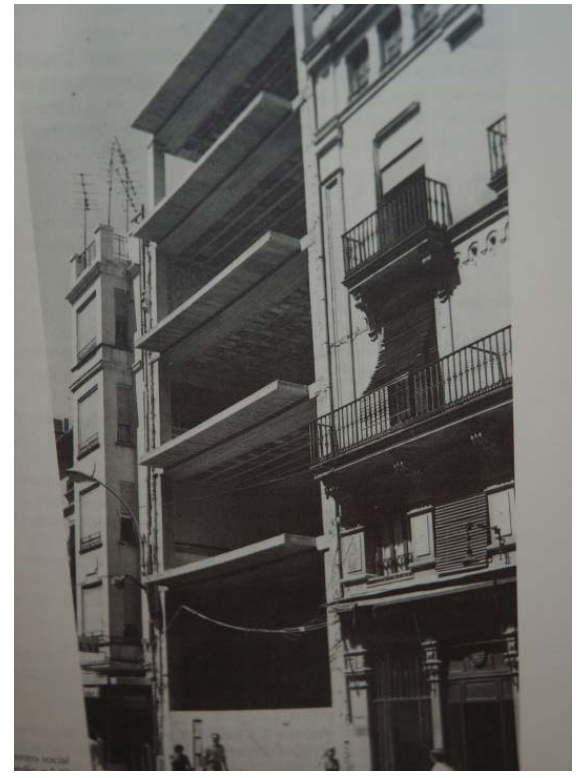

Figura 1. Sede social, calle Enmedio

de D. Roberto Follía el 16 de diciembre de 1969 por el precio de 9100000 pesetas, pagadas al contado y libre de inquilinos. En 1971 se llegó al acuerdo por el consejo rector de derribar el edificio existente y construir un nuevo centro social. Se encargó el proyecto y la dirección de obra al arquitecto D. Vicente Traver González Espresati y designando aparejador a D. Ernesto Guaita. La construcción (figura 1) fue realizada en un principio por la empresa de D. Vicente Guillamón y acabada por D. Domingo Martí de la ciudad de Nules.

La obra se inauguró el día 30 de enero de 1974, presidiendo el acto el jefe provincial del movimiento y gobernador de la ciudad $\mathrm{D}$. Juan de Aizpurúa y la bendijo el obispo de la diócesis D. José María Cases, siendo presidente de la entidad D. José Pachés Marí.

En 1938, durante el periodo de incautación de la Cooperativa Agrícola por el comité del Frente Popular, el domicilio social y posterior sede la Caja Rural de Crédito se fija en la calle Largo Caballero, n. 8 (después José Antonio y en la actualidad Gasset), que nacieron en un espacio muy reducido en el que apenas cabían tres empleados y, aunque fue ampliándose con unos pocos metros más, nunca llegó a ser una oficina que atrajera a los clientes, por la sensación de agobio que producía; téngase en cuenta, por ejemplo, que el despacho del director general estaba ubicado en una habitación sin luz natural ni ventilación (figura 2).

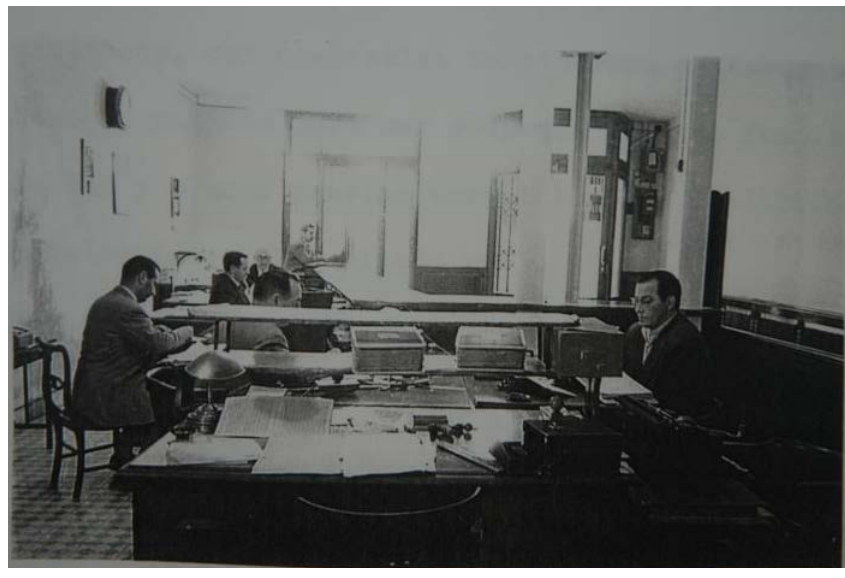

Figura 2. Antiguas oficinas, calle Gasset

El 20 de enero de 1946, reunidos en junta general extraordinaria y al amparo de la ley de Cooperación de 2 de enero de 1942, se acuerda constituir la Caja Rural de la Cooperativa Agrícola San Isidro de Castellón. Esta constitución significa realmente la adaptación de la cooperativa a una nueva legalidad, siendo nombrado su primer presidente D. Domingo Traver Escrig y su primer director general D. Antonio Dols Sacristán, con un capital de 453000 pesetas y unas imposiciones de 3500000 de pesetas.

Durante el primer trimestre de 1957, se publica en el Boletín Oficial de la Provincia el anuncio de pública subasta de la Audiencia Provincial, propiedad del Excmo. Ayuntamiento de Castellón, sita en la calle Gasset n. ${ }^{\circ} 1$ con una superficie de $301 \mathrm{~m}^{2}$.

Se acudió a la subasta, que salía por un precio de 2500000 pesetas, presentando una plica por importe de 2500001 pesetas, que fue la adjudicataria, si bien no se podía tomar posesión del inmueble hasta que no estuviera construido el nuevo Palacio de Justicia (figura 3), dependiente del Ministerio de la misma. Si bien el Ayuntamiento satisfacería a la Caja Rural un interés del $4 \%$ anual hasta su total desalojo, que ocurrió en mayo de 1974.

Durante el año 1959, se adquirieron sucesivamente las tres casas colindantes en las calles Vera e Isaac Peral, por un precio de 1697000 pesetas, debiendo pagar además 763000 pesetas a los inquilinos de las mismas para su desalojo. 


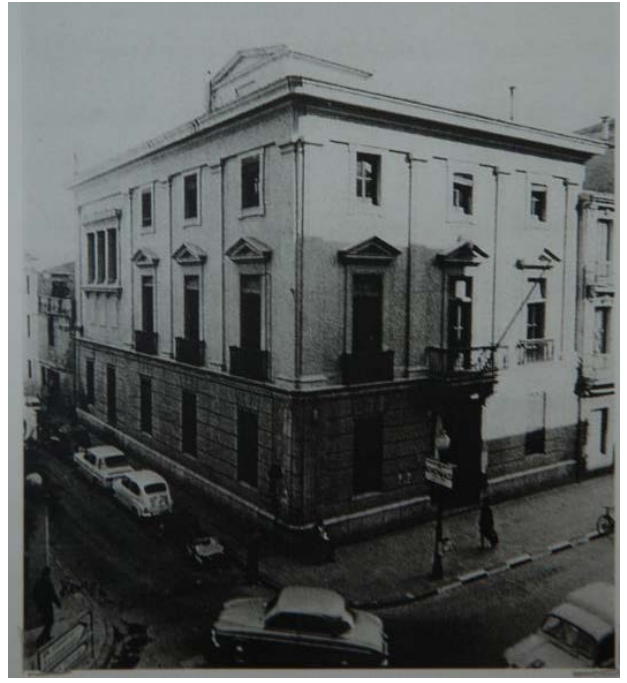

Figura 3. Edificio Audiencia Provincial.

El 12 de marzo de 1978 se realizaron las primeras gestiones para la edificación de la sede central de la caja, encargándose el proyecto al arquitecto D. Vicente Traver González Espresati y como aparejador a D. Ernesto Guaita. Se proyectó un edificio de seis plantas, reservándose la caja el sótano, planta baja y entresuelo, permutando el coste por el vuelo, las obras del sótano, estructura y tabiquería y dinero efectivo, adjudicándose la edificación la empresa Luis Batalla S. A.

Se inauguró la obra el 27 de febrero de 1980, siendo presidente de la caja D. Juan Barreda, asistiendo el gobernador civil, D. Rafael Montero, el presidente de la Diputación, D. Joaquín Farnós y los delegados de Agricultura y Trabajo, D. Eladio Cheza y D. Enrique Ojea, respectivamente. Bendijo las obras el Rvdo. Gil Roger Roger, canónigo de Segorbe y consiliario de la Caja Rural Provincial, siendo di-

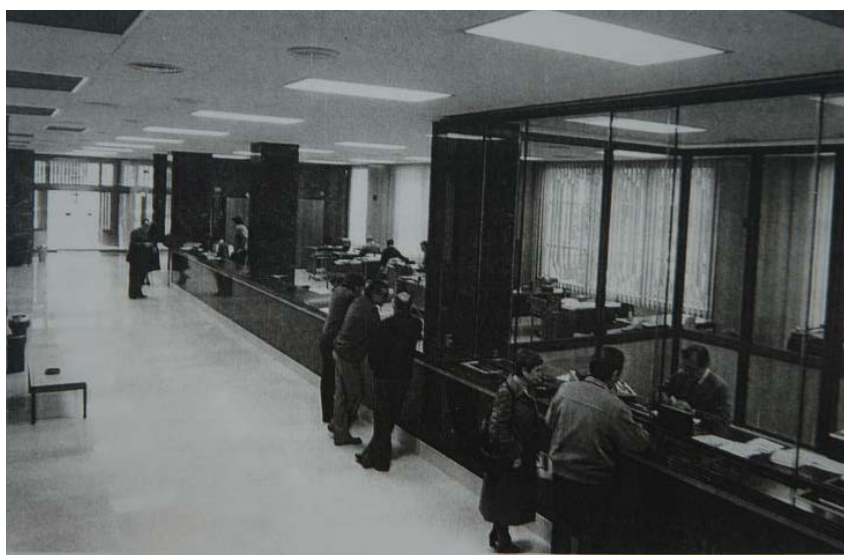

Figura 4. Interior de las oficinas en la calle Gasset rector de esta última D. Ramón Oñate. En la figura 4 puede observarse el interior de las oficinas.

En el año 1956, habitaban en Castellón unas 55000 personas $y$, aunque era una ciudad pequeña y con distancias cortas, había un barrio (el raval de San Félix) que, tradicionalmente, desde la fundación del Gremio de Agricultores, tenía una amplia adhesión a la Caja Rural y, aunque el trayecto era corto hasta la calle José Antonio (hoy Gasset), el consejo rector tenía la preocupación de acercar en lo posible sus oficinas a estos habitantes, que siempre se habían mantenido fieles a la Caja Rural; por ello, con muy buen criterio y para dicho fin, se acordó adquirir un edificio para la ubicación de una sucursal en dicho raval.

Por ello, se encargó al agente de la propiedad D. Vicente Hernández Cosín la búsqueda y acuerdos para localizar esta primera sucursal, adquiriéndose un edificio de 52,10 $\mathrm{m}^{2}$ ubicado en el chaflán de la plaza Clavé, calles S. Félix y Conde Pestagua. La escritura de compra se firmó el 24 de febrero de 1956 por el precio de 525000 pesetas y pagando una indemnización por desalojo al inquilino de la planta baja de 150000 pesetas.

De realizar las obras de acondicionamiento del edificio se encargó el arquitecto D. Vicente Traver Tomás. Con ello se apertura la primera oficina urbana de la Caja Rural San Isidro, siendo esta la primera sucursal que se abriera entre todas las entidades de crédito y ahorro presentes en Castellón, marcando con ello un hito, que después fue copiado por todas las demás entidades.

Años más tarde, el 11 de febrero de 1966, con el fin de ampliar la superficie, se adquirió el local colindante, de nueva edificación, de $65,50 \mathrm{~m}^{2}$ por el precio de 400000 pesetas, ampliándose así el reducido espacio de los locales anteriores. En este nuevo edificio se ubicó el consejo rector de la caja, dado que en las oficinas de la calle José Antonio n. ${ }^{\circ} 8$, era imposible mantenerlo por falta de espacio.

\section{En el cultivo del arroz en Castellón}

En la década de los años 50, estaba en pleno auge el cultivo del arroz; no se notaba en ningún momento la falta de mano de obra. El agua todavía cubría con suficiencia las necesidades agrarias. 
El minifundismo que existía en la zona arrocera, con una extensión aproximada de 10000 hanegadas, era, como lo fue siempre un problema tremendamente agudo.

De este problema y, en general, del cultivo del arroz en España, el Profesor Tamames, en su libro Estructuras Económicas de España dice:

La exportación española del arroz, solo es posible a precios muy bajos, pues se ha de competir con las ofertas de los grandes países exportadores de este cereal, típicamente subdesarrollados que cuentan por consiguiente con una mano de obra abundante y muy barata (Birmania, Tailandia, etc.) o que tienen precios subvencionados por sus gobiernos (EE.UU.).

Nuestras exportaciones se dirigen principalmente a Extremo Oriente y no constituyen ciertamente un negocio del que podamos estar orgullosos, pues los precios que se obtienen son muy inferiores a los del mercado interior y solo pueden sostenerse merced a la prima que de hecho pagan los consumidores nacionales. En estas condiciones hay que plantearse seriamente si no convendría más reducir el área de arrozales transformándolos en cultivos más rentables, que no seguir manteniendo su cultivo en su actual extensión.

En el termino municipal de Castellón existían 992 fincas dedicadas al cultivo de este cereal; de ellas, 640 no pasaban de una superficie de 5 hanegadas, 202 fincas comprendían entre 5 y 10 hanegadas y 150 eran mayores de 11 hanegadas, lo que quiere decir que el $84,9 \%$ de la superficie destinada al cultivo del arroz adolecía de un defecto estructural, siendo inapropiada para ser cultivada racionalmente.

La cooperativa nunca había intervenido con sus servicios en la zona arrocera. Después de un estudio sobre las necesidades, se concretó en principio la conveniencia de tener un secadero propio, ya que muchos socios carecían de él.

Examinada la zona, se localizó una parcela junto al camino Serradal (figura 5) de una extensión de 6 hanegadas y 102 brazas, propiedad de D. Vicente Balaguer y que, una vez realizadas las negociaciones, se compró por 500000 pesetas. La firma de la escritura se realizó el 20 de noviembre de 1958 ante el notario D. Antonio Campesino e inmediatamen-

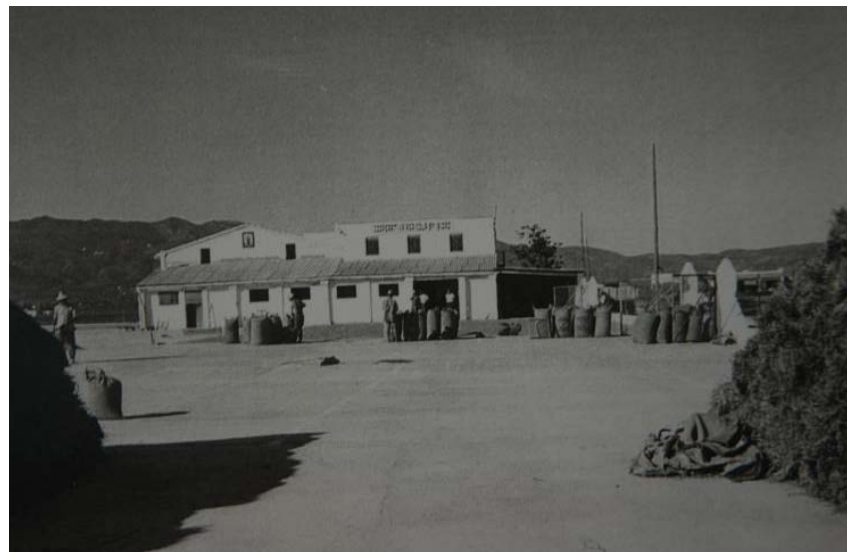

Figura 5. Secadero de arroz en el camino del Serradal

te se emprendieron los trabajos de construcción, llevándose a cabo por el contratista Manuel Torrella y Cía. Los trabajos consistieron principalmente en el levantamiento de un murete por los lindes de la finca y en su relleno con piedras, elevando así el suelo sobre el nivel de la carretera, asegurando así las cosechas esparcidas ante una posible inundación por lluvias. La superficie tenía un gradiente suficiente para desalojar las aguas de lluvia. La finca se señalizó en parcelas de superficie suficiente para el secado en cada una de ellas de $100 \mathrm{~kg}$ de arroz con cáscara para su secado.

Se adquirieron dos trilladoras de la marca Vert120, metálicas, construidas en Torroella de Montgrí (Girona) en el año 1957 y el 17 de junio de 1959 por un precio de 165847 y 205035 pesetas respectivamente.

Una trilladora quedaría emplazada fija en el secadero (figura 6). Se alimentaba con las grabas de arroz, previamente depositadas mediante una

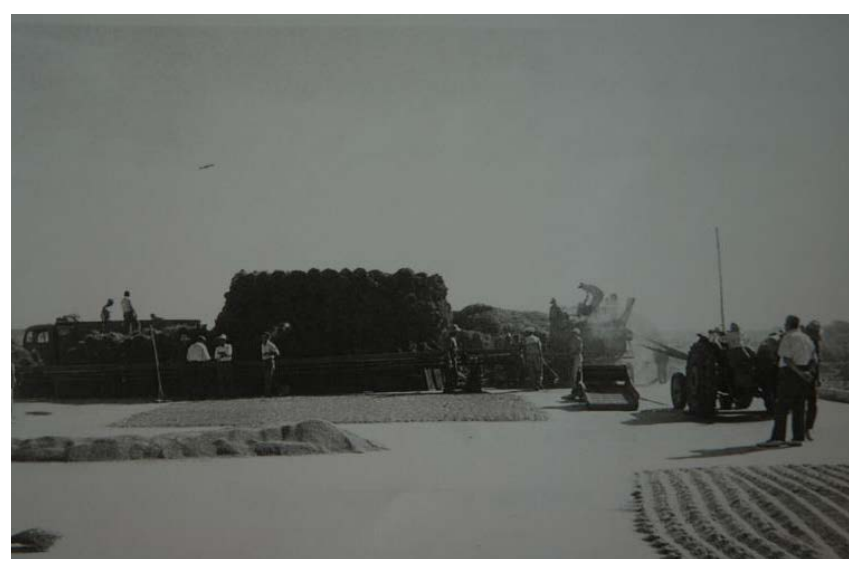

Figura 6. Operaciones de trilla del arroz 


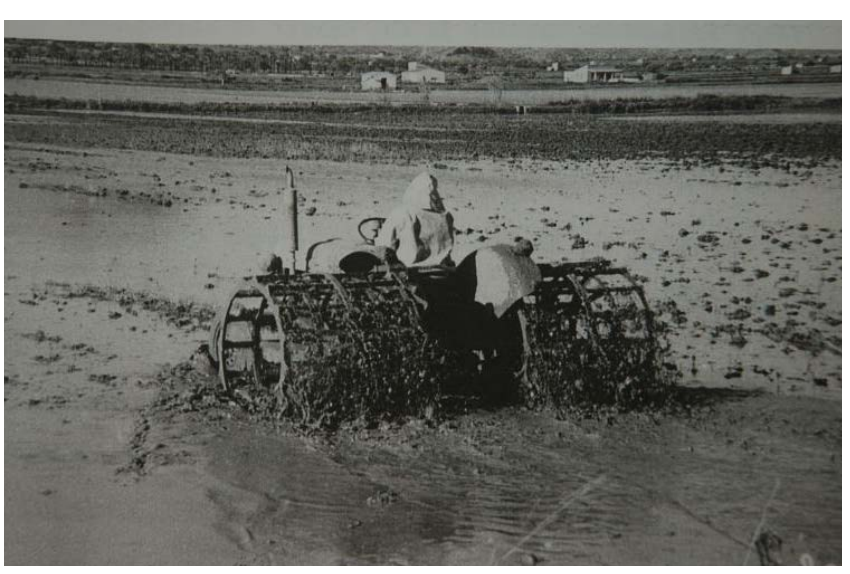

Figura 7. Tractor para el fangueo del arroz

larga cinta transportadora de $40 \mathrm{~m}$ accionada por motor de $4 \mathrm{hp}$ que se adquirió el 12 de setiembre de 1960 por un precio de 91513 pesetas. Esta trilladora estaba accionada por un tractor Honomag-Barreiros de $60 \mathrm{hp}$ y otro de $40 \mathrm{hp}$ de la marca Ebro.

La trilladora que circulaba entre los secaderos de los socios en un itinerario rotativo fijado de antemano era accionada por un tractor Fordson.

Pasados unos años, en 1961, al aparecer las cosechadoras, se pensó en adquirir una de esas máquinas, aunque con algunas dudas, dada la existencia de algunas fincas con un terreno excesivamente turboso. Finalmente, se adquirió en setiembre de 1961 una cosechadora pequeña, marca SantanaClaya, con motor Land Rover de $45 \mathrm{hp}$, por el precio de 545128 pesetas, que resultó un fracaso. Solo llegó a trabajar algunas horas en varias campañas, pasando después al retiro y posterior venta.

Para el fangueo, los tractores Fordson, Ebro y Dexta realizaron, con dos tractoristas por unidad y en jornadas de trabajo de 14 horas, el fangueo que los socios demandaban (figura 7).

En el secadero del Serradal, se almacenaban y se suministraban los abonos adecuados para cada época del abonado del arroz. Se suministraba a los socios el sulfato amónico, el superfosfato de cal y el sulfato de potasa que se precisaba.

En 1969, el Ayuntamiento de Castellón patrocinó la desecación de las tierras con ayuda estatal, construyendo acequias de drenaje e iniciando una campaña de desinsectación que duró hasta el año 1974. Se suponía que a su cultivo se debía el notable nú- mero de enfermos del paludismo, dado que las zonas pantanosas eran idóneas para el crecimiento y desarrollo del mosquito Anopheles, cuya hembra transmitía la enfermedad con su picadura pese a la existencia de peces autóctonos como el samarruc o los importados desde Australia del género gambusia que son comedores de larvas, eran insuficientes para contener el número de mosquitos y de la enfermedad, con lo que el cultivo del arroz dejó de existir en Castellón.

\section{Obra social a favor de los labradores}

No era fácil encontrar en 1963 viviendas que se localizaran en lugares céntricos con aceptables condiciones de habitabilidad, a precios asequibles y en condiciones de pago al alcance de todos, sobre todo para los agricultores de renta media-baja.

Por ello, la Caja Rural S. Isidro se comprometió como obra social, a edificar 126 viviendas de protección oficial y un almacén en los bajos para uso de la cooperativa. Por ello, entre 1959 y 1960 se compraron seis solares, sitos en Hermanos Bou, Bellver y Prim que, en conjunto, ocupaban una superficie de $2920,70 \mathrm{~m}^{2}$ por un importe de 4984496 pesetas. La junta rectora acordó la construcción de las mismas, encargándole la redacción del proyecto al arquitecto D. Vicente Traver González Espresati.

La obra comprendía la construcción de 108 viviendas de tres habitaciones dormitorio, comedor-salón, cocina, servicios y terraza y de 18 viviendas de mayor superficie que incluían una cuarta habitación dormitorio.

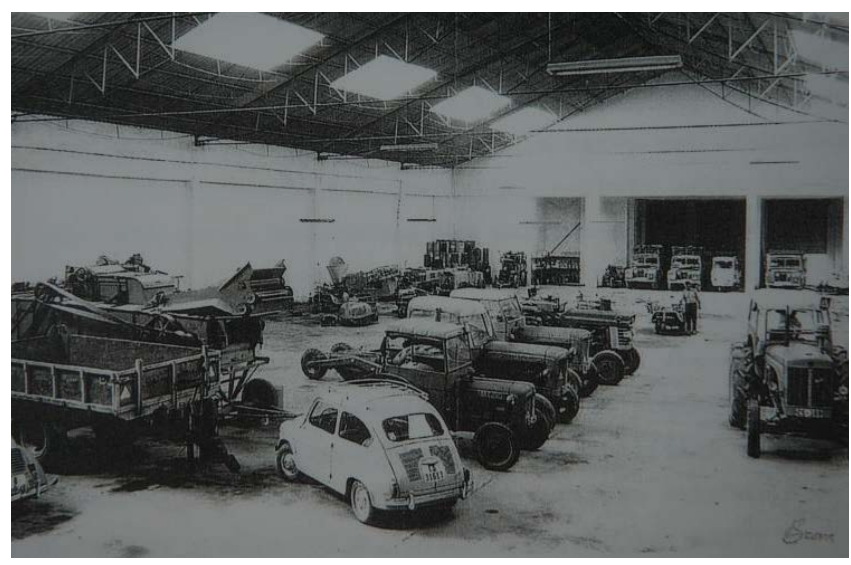

Figura 8. Almacén de la cooperativa S. Isidro en los bajos de las viviendas de la obra social 


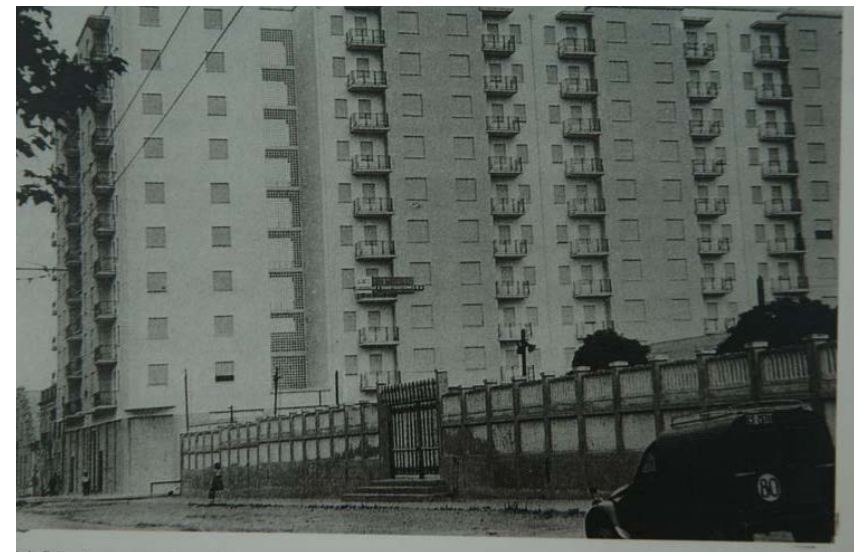

Figura 9. Viviendas de la obra social

En los bajos se edificaría un almacén con locales exteriores a tres calles y un sótano de $1000 \mathrm{~m}^{2}$ para garaje con entrada y salida por las calles Hermanos Bou y Prim, respectivamente (figura 8).

La cooperativa ocupó los bajos sin pagar alquiler alguno a la caja, que utilizó como parque de maquinaria, que atendía a los servicios que prestaba la cooperativa en sus actividades. La obra la llevo a cabo la empresa Dragados y Construcciones S. A. El coste de la obra fue de 31187231 pesetas

El día 20 de abril de 1964 se acordaron las normas para adjudicar las viviendas entre los socios e im- positores solicitantes, que debían reunir unas condiciones de necesidad manifiesta por carecer de vivienda propia.

En el salón de asambleas de la caja central, ubicado en el tercer piso de la calle Ruiz Zorrilla, antes Caja Rural Provincial, posteriormente Ruralcaja y hoy Cajamar, e inicialmente Hotel Suizo, se celebró el acto de adjudicación por sorteo.

Días después se celebraron los contratos privados de compra-venta y la forma de pago, que consistía en el $30 \%$ de su precio al contado y el resto mediante un préstamo a un plazo de 15 años y a un interés del $5 \%$. Se ha de hacer hincapié en el largo plazo de financiación ofrecido a los compradores, inusual para aquella época, dadas las limitaciones que en aquellos años imponía el Banco de España para estos plazos de amortización.

El 2 de julio de 1964 se acordó otorgar la escritura de declaración de obra nueva y de división horizontal y el 19 de diciembre del mismo año se obtendría la calificación definitiva (figura 9).

En el mes de noviembre de 1970, se procedió a las correspondientes escrituras de compra-venta ante los diferentes notarios de la ciudad.

\section{Referencias}

Antonio Dols Sacristán. Cifra y hechos relevantes Caja Rural S. Isidro 1948-1986.

Varios Autores. Centenario de la Cooperativa Agrícola San Isidro y Caja Rural Castellón. 